Brit. F. vener. Dis. (1971) 47, 407

\title{
Inhibition of growth of treponemes by antimicrobial agents
}

\section{J. ABRAMSON AND R. M. SMIBERT*}

Anaerobe Laboratory, Virginia Polytechnic Institute, and State University, Blacksburg, Virginia 24060, U.S.A.

The antimicrobial sensitivity of treponemes has been little investigated in comparison with that of micro-organisms that can be more easily cultured. Results of antibiotic sensitivity tests in vitro have been reported for oral treponemes in which inhibitory concentrations were determined by observing the growth of cultures (Fitzgerald and Hampp, 1952; Berger, 1956, 1958a, b, c, 1959, 1960; Hampp and Fitzgerald, 1959; Berger and Marggraf, 1960). Eagle and Musselman (1944) reported that a penicillin concentration of 0.1 to $0.25 \mathrm{units} / \mathrm{ml}$. rendered 90 to 99 per cent. of Reiter treponemes non-viable in 8 to $12 \mathrm{hrs}$.

Inhibition of motility has been used to determine the sensitivity of living suspensions of Treponema pallidum and other pathogenic treponemes to antibiotics (Dunham, Hamre, McKee, and Rake, 1944; Nelson, 1948; Nell, 1954). The sensitivity of the nonpathogenic cultivable Nichols, Kazan, and Reiter treponemes to erythromycin was determined by observing the inhibition of motility of cells under darkfield microscopy (Keller and Morton, 1953).
Penicillin has been the accepted treatment for syphilis since the early 1940s (Mahoney, Arnold, and Harris, 1943; Willcox, 1954; Yobs, Clark, Mothershed, Bullard, and Artley, 1968), but virulent $T$. pallida have recently been recovered from man and animals treated with presumably adequate concentrations of penicillin (Collart, Borel, and Durel, 1962; Yobs and others, 1968).

Until such time as the pathogenic species have been cultured in vitro, cultivable treponemes must serve as a model for determining the effect of antibiotics. The purpose of the present investigation was to determine the concentrations of antibiotics an $\$$ other antimicrobial agents that inhibited growth of representative strains of various species of cultivable treponemes.

\section{Material and methods}

TREPONEME STRAINS

The strains used in these studies are listed in Table I.

†Bellco Glass, Inc., P.O. Box B, Vineland, New Jersey 08360,

U.S.A.

$\ddagger$ Pfizer Diagnostics Division, New York, New York 10036, U.S.A. §Difco Laboratories, Detroit, Michigan 48201, U.S.A.

Diacetyl tartaric acid ester of tallow monoglycerides, Witco Chemical Co. 277 Park Avenue, New York, New York 10017, U.S.A.

Received for publication
$\star$ Requests for reprints

TABLE I Treponemes studied

\begin{tabular}{|c|c|c|c|c|}
\hline Old name & New name & Origin & Source & Special requirements \\
\hline $\begin{array}{l}\text { Reiter } \\
\text { Kazan } \\
T . \text { refringens } \\
T . \text { calligyrum } \\
\text { Nichols (avirulent) }\end{array}$ & $\begin{array}{l}T . \text { phagedenis } \\
T . \text { phagedenis } \\
T . \text { refringens } \\
T . \text { refringens } \\
T \text {. refringens }\end{array}$ & Genital-anal & $\begin{array}{l}\mathrm{CDC}^{\mathrm{b}} \\
\mathrm{CDC} \\
\mathbf{P c} \\
\mathbf{P} \\
\mathrm{CDC}\end{array}$ & Rabbit serum \\
\hline $\begin{array}{l}T . \text { microdentium }(\mathrm{FM}) \\
T . \text { ambiguum } \\
\mathrm{T}-32-\mathrm{A} \\
T . \text { vincentii }(\mathrm{N}-9)\end{array}$ & $\begin{array}{l}T . \text { denticola } \\
T . \text { denticola } \\
T . \text { denticola } \\
T . \text { vincentii }\end{array}$ & Human oral cavity & $\begin{array}{l}\text { NIH }^{d} \\
\text { P } \\
\text { Dr. T. Rosebury } \\
\text { NIH }\end{array}$ & Rabbit serum cocarboxylase \\
\hline $\begin{array}{llr}\text { Human oral } & \text { (HO) } & 5 \\
& \text { HO } & 18 \\
& \text { HO } & 27\end{array}$ & $-^{a}$ & Human oral cavity & VPI ${ }^{e}$ & Rumen fluid \\
\hline $\begin{array}{lll}\text { Pig faeces } & \text { (PF) } & 23 \\
& \text { PF } & 31 \\
& \text { PF } & 39 \\
& \text { PF } & 28 \\
& \text { PF } & 44\end{array}$ & $\begin{array}{l}- \\
- \\
-\end{array}$ & Pig faeces & VPI & Rumen fluid \\
\hline
\end{tabular}

Species not designated. bVenereal Disease Research Laboratory, Center for Disease Control. cPasteur Institute (Paris). ${ }^{\mathrm{d} I n s t i t u t e ~ o f ~ D e n t a l ~ H e a l t h ~}$ National Institutes of Health. eVirginia Polytechnic Institute and State University, Anaerobe Laboratory. 
Stock cultures were incubated 24 to $48 \mathrm{hrs}$. before inoculation into test media. Cell counts after incubation were $10^{7}$ to $10^{8}$ treponemes $/ \mathrm{ml}$., using a Petroff-Hausser Counting Chamber. Viable cell counts, using 10-fold dilutions of cultures, agreed with the microscopic cell counts. A stock culture was inoculated into $7.5 \mathrm{ml}$. pre-reduced test medium, giving a final cell count of $10^{5}$ to $10^{6}$ treponemes $/ \mathrm{ml}$. All cultures were incubated at $37^{\circ} \mathrm{C}$. except $T$. vincentii which was incubated at $34^{\circ} \mathrm{C}$.

\section{MEDIA}

Pre-reduced, anaerobic media with an oxidation-reduction potential of less than $-150 \mathrm{mv}$. were prepared as described in the 'Outline of Clinical Methods in Anaerobic Bacteriology' (Anaerobe Laboratory, 1970). All inoculations were carried out under $\mathrm{O}_{2}$-free nitrogen using the V.P.I. Anaerobic Culture System. $\dagger$

Treponemes requiring serum were cultured in PYGS medium which contained peptone $M \ddagger, 2 \mathrm{~g}$.; yeast extract $\S$ $1 \mathrm{~g}$; ; dextrose $1 \mathrm{~g}$.; agar $0.2 \mathrm{~g}$; ; ammonium sulphate 0.05 g.; soluble starch $\$ 0.05$ g.; TEM-4T $\| 0.016$ g.; L-cysteine $\mathrm{HCl}$ hydrate $0.16 \mathrm{~g}$; ; sodium bicarbonate $0.5 \mathrm{~g}$.; resazurin solution $(25 \mathrm{mg}$. per cent.) $0.4 \mathrm{ml}$.; salt solution $50 \mathrm{ml}$. (anhydrous $\mathrm{MgSO}_{4} 0.02 \mathrm{~g}$; $\mathrm{CaCl}_{2}$. $2 \mathrm{H}_{2} \mathrm{O} 0.02$ g.; $\mathrm{K}_{2} \mathrm{HPO}_{4} 0.1$ g.; $\mathrm{KHPO}_{4} 0.1$ g.; $\mathrm{NaCl}$ $0.2 \mathrm{~g}$. dissolved in $100 \mathrm{ml}$. distilled water); and distilled water $50 \mathrm{ml}$. The $\mathrm{pH}$ was adjusted to 6.5 before autoclaving and was 6.8 to 7.4 after sterilization. Sterile rabbit serum and cocarboxylase were added aseptically to autoclaved medium to final concentrations of 12 and 0.00012 per cent. respectively.

\section{RABBIT SERUM}

This was sterilized by filtration through a $0.45 \mu \mathrm{m}$. pore diameter membrane filter, aseptically dispensed into sterile glass bottles, heat inactivated at 58 to $60^{\circ} \mathrm{C}$. for $4 \mathrm{hrs}$., and stored at $-20^{\circ} \mathrm{C}$.

\section{COCARBOXYLASE}

$50 \mathrm{mg}$. were dissolved in $100 \mathrm{ml}$. distilled water and filtered through a $0 \cdot 22 \mu \mathrm{m}$. pore diameter membrane filter; $1 \mathrm{ml}$. of the sterile solution was added to $50 \mathrm{ml}$. sterile inactivated rabbit serum.

Treponemes requiring rumen fluid were cultured in human oral (HO) medium which consisted of peptone $M$ $0.1 \mathrm{~g}$.; yeast extract $0.1 \mathrm{~g}$.; dextrose $0.5 \mathrm{~g}$.; agar $0.2 \mathrm{~g}$.; ammonium sulphate $0.05 \mathrm{~g}$; soluble starch $0.05 \mathrm{~g}$.; Lcysteine- $\mathrm{HCl} 0.16 \mathrm{~g}$; ; sodium bicarbonate 0.5 per cent.; resazurin solution $0.4 \mathrm{ml}$; salt solution $50 \mathrm{ml}$.; rumen fluid $28 \mathrm{ml}$; and distilled water $22 \mathrm{ml}$. The $\mathrm{pH}$ was adjusted to 6.5 before autoclaving and was 6.8 to 7.4 after autoclaving. These cultures were maintained in E-medium, which consisted of peptone $M 0.05 \mathrm{~g}$.; yeast extract 0.05 g.; dextrose 0.14 g.; agar 0.2 g.; ammonium sulphate $0.05 \mathrm{~g}$.; soluble starch $0.05 \mathrm{~g}$.; L-cysteine- $\mathrm{HCl} 0.16 \mathrm{~g}$; sodium bicarbonate $0.5 \mathrm{~g}$; resazurin solution $0.4 \mathrm{ml}$.; salt solution $50 \mathrm{ml}$; rumen fluid $28 \mathrm{ml}$; and distilled water $22 \mathrm{ml}$. The $\mathrm{pH}$ was adjusted to 6.5 before sterilizing and was 6.8 to 7.4 after sterilizing.

\section{RUMEN FLUID}

Contents from the rumen of cattle were collected and filtered through two layers of cheesecloth. The liquid portion was siphoned into bottles, autoclaved at $121^{\circ} \mathrm{C}$. at $15 \mathrm{lb}$. pressure for $15 \mathrm{~min}$. and stored at $4^{\circ} \mathrm{C}$. In preparing media, the rumen fluid was centrifuged at $10,000 \mathrm{G}$. for $20 \mathrm{~min}$., added to the media, and sterilized by autoclaving.

All treponemes were grown in pre-reduced E-medium or in pre-reduced E-medium with 12 per cent. serum and 0.00012 per cent. cocarboxylase for 2 to 3 days and preserved at $-85^{\circ} \mathrm{C}$.

\section{ANTIMICROBIAL AGENTS}

All antibiotics and chemicals were diluted so that the final concentrations in $7.5 \mathrm{ml}$. culture medium were 0.01 , $0 \cdot 1,1,10,100,500$, and $1,000 \mu \mathrm{g}$. or units $/ \mathrm{ml}$.

TABLE II Growth inhibition of treponemes by penicillins

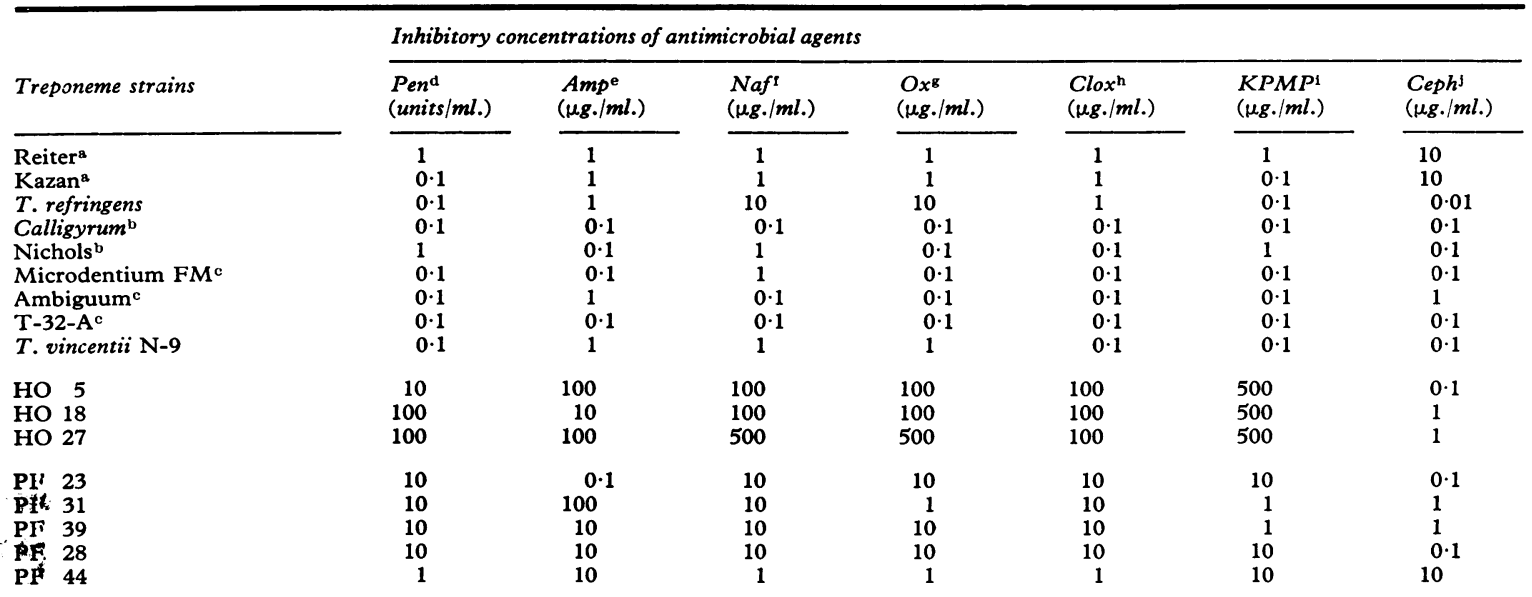

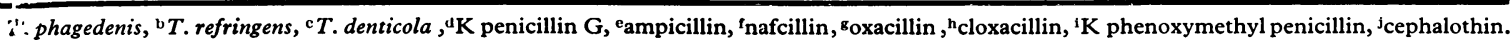


BACTERIOSTASIS

The bacteriostatic concentration of an antimicrobial agent was considered to be the lowest concentration in the dilution series in which no growth was observed after 3 days. All cultures were examined for visible growth and were also examined with a darkfield microscope ( $\times 1,000)$ on the 3rd day of incubation. Bacteriostatic activity was assessed by comparing the growth, cell morphology, and motility of treponemes in media containing the antimicrobial agent with those of treponemes in control media.

\section{Results}

Tables II to VI show the concentrations of antimicrobial agents that inhibited the growth of the treponemes studied. They were all apparently sensitive to cephalothin and most strains were apparently sensitive to the penicillin group of antibiotics except the human oral (HO) isolates (Table II).

Table III shows that all strains were apparently sensitive to bacitracin and erythromycin while most strains were sensitive to tylosin, lincomycin, and vancomycin. Treponeme strains varied in their sensitivity to novobiocin.

In Table IV, the tetracycline group of antibiotics were inhibitory to all treponemes in low concentrations except the strains isolated from pig faeces (PF-group). Treponemes were resistant to chloramphenicol.

Growth inhibitory concentrations of other antimicrobial agents to individual strains of treponemes were varied and are listed in Tables V and VI.

TABLE III Growth inhibition of treponemes by macrolides and other antibiotics

\begin{tabular}{|c|c|c|c|c|c|c|}
\hline \multirow[b]{2}{*}{ Treponeme strains } & \multicolumn{6}{|c|}{ Inhibitory concentrations of antimicrobial agents } \\
\hline & $\begin{array}{l}\text { Novod } \\
(\mu g . / m l .)\end{array}$ & $\begin{array}{l}\text { vanco } \\
(\mu g . / m l .)\end{array}$ & $\begin{array}{l}B a c^{\mathrm{p}} \\
(\mu g . / m l .)\end{array}$ & $\begin{array}{l}\text { Eryg } \\
(\mu g . / m l .)\end{array}$ & $\begin{array}{l}T y l o^{\mathrm{h}} \\
(\mu \mathrm{g} . / \mathrm{ml} .)\end{array}$ & $\begin{array}{l}\text { Linco }^{1} \\
(\mu \mathrm{g} . / \mathrm{ml} .)\end{array}$ \\
\hline Reiter $^{\mathbf{a}}$ & 100 & 1 & 1 & $0 \cdot 1$ & 100 & 100 \\
\hline Kazan $^{\mathrm{a}}$ & 10 & 1 & $0 \cdot 1$ & $0 \cdot 1$ & 10 & 10 \\
\hline T. refringens & 100 & 1 & 0.01 & 0.01 & 10 & 1 \\
\hline Calligyrum $^{\mathrm{b}}$ & 500 & 1 & $0 \cdot 1$ & $0 \cdot 1$ & 10 & 1 \\
\hline Nichols ${ }^{\mathrm{b}}$ & 500 & $0 \cdot 1$ & $0 \cdot 1$ & 0.01 & 1 & $\hat{1}$ \\
\hline Microdentium FMc & 100 & 1 & 1 & 0.01 & 100 & 10 \\
\hline Ambiguum $^{c}$ & 10 & 1 & 1 & $0 \cdot 1$ & 10 & 1 \\
\hline$T-32-A^{c}$ & 10 & 10 & $0 \cdot 1$ & 0.01 & 10 & 10 \\
\hline T. vincentii N-9 & 1 & 1 & $0 \cdot 1$ & $0 \cdot 1$ & 1 & 10 \\
\hline $\mathrm{HO} 5$ & 100 & 1 & 1 & 0.01 & 1 & 10 \\
\hline HO 18 & 500 & 10 & 1 & 0.01 & 1 & 10 \\
\hline HO 27 & 100 & 10 & 1 & 0.01 & 1 & 10 \\
\hline PF 23 & $0 \cdot 1$ & 100 & 10 & $0 \cdot 1$ & 10 & 10 \\
\hline PF 31 & 10 & 100 & 10 & 0.1 & 10 & 10 \\
\hline PF 39 & 1 & 500 & 10 & $0 \cdot 1$ & 10 & 10 \\
\hline PF 28 & 1 & 100 & 1 & $0 \cdot 1$ & 10 & 10 \\
\hline PF 44 & 10 & 100 & 1 & $0 \cdot 1$ & 10 & 10 \\
\hline
\end{tabular}

${ }^{\mathrm{a} T}$. phagedenis, ${ }^{\mathrm{b}} T$. refringens, ${ }^{\mathrm{c}} T$. denticola, "dnovobiocin, ${ }^{\mathrm{e}}$ vancomycin, $\mathrm{f}$ bacitracin, $\mathrm{g}$ erythromycin, ${ }^{\mathrm{h}}$ tylosin, ${ }^{\mathrm{i}} \mathrm{lincomycin}$.

TABLE IV Growth inhibition of treponemes by the tetracyclines and chloramphenicol

\begin{tabular}{|c|c|c|c|c|c|c|c|}
\hline \multirow[b]{2}{*}{ Treponeme strains } & \multicolumn{7}{|c|}{ Inhibitory concentrations of antimicrobial agents } \\
\hline & $\begin{array}{l}T_{e t}^{\mathrm{d}} \\
(\mu g . / m l .)\end{array}$ & $\begin{array}{l}\text { Chlortet } \\
(\mu g . / m l .)\end{array}$ & $\begin{array}{l}\text { Oxytet } \\
(\mu \mathrm{g} . / \mathrm{ml} .)\end{array}$ & $\begin{array}{l}\text { Dmethchg } \\
(\mu g . / m l .)\end{array}$ & $\begin{array}{l}D o x y^{\mathrm{h}} \\
(\mu g . / m l .)\end{array}$ & $\begin{array}{l}\text { Methac } \\
(\mu \mathrm{g} . / \mathrm{ml} .)\end{array}$ & $\begin{array}{l}\text { Chloram } \\
(\mu \mathrm{g} . / \mathrm{ml} .)\end{array}$ \\
\hline Reiter $^{\mathbf{a}}$ & 1 & 1 & 10 & 1 & 1 & 1 & 500 \\
\hline Kazan $^{\mathrm{a}}$ & 1 & 10 & 10 & 1 & $0 \cdot 1$ & 1 & 500 \\
\hline$T$. tefringens & 1 & 10 & 1 & 1 & $0 \cdot 1$ & $0 \cdot 1$ & 100 \\
\hline Calligyrumb & 1 & 10 & 1 & 1 & 1 & $0 \cdot 1$ & 100 \\
\hline Nichols ${ }^{b}$ & 1 & 10 & 1 & 1 & 1 & 1 & 100 \\
\hline Microdentium FMc & 1 & 10 & 1 & 1 & 1 & 1 & 100 \\
\hline Ambiguum ${ }^{c}$ & 1 & 10 & 10 & $0 \cdot 1$ & $0 \cdot 1$ & $0 \cdot 1$ & 100 \\
\hline$T-32-A^{c}$ & 1 & 1 & 1 & $0 \cdot 1$ & $0 \cdot 1$ & $0 \cdot 1$ & 100 \\
\hline T. vincentii N-9 & 1 & 1 & 1 & $0 \cdot 1$ & $0 \cdot 1$ & 0.1 & 100 \\
\hline HO 5 & 10 & 10 & 10 & 10 & 10 & 1 & 100 \\
\hline HO 18 & 1 & 10 & 1 & 1 & 1 & 1 & 100 \\
\hline HO 27 & 1 & 10 & 1 & 1 & 1 & 1 & 100 \\
\hline PF 23 & 100 & 500 & 500 & 100 & 100 & 100 & 100 \\
\hline PF 31 & 100 & 100 & 100 & 100 & 100 & 100 & 100 \\
\hline PF 39 & 100 & 500 & 100 & 100 & 100 & 100 & 100 \\
\hline PF 28 & 100 & 100 & 100 & 100 & 500 & 500 & 10 \\
\hline PF 44 & 500 & 500 & 500 & 100 & 500 & 100 & 100 \\
\hline
\end{tabular}

a $T$. phagedenis, "T. refringens, " $T$. denticola, dtetracycline, echlortetracycline, foxytetracycline, ${ }^{g}$ demethylchlortetracycline, ${ }^{\mathrm{h}}$ doxycycline, ${ }^{\mathrm{i}}$ methacycline, jchloramphenicol. 
TABLE V Growth inhibition of treponemes by aminoglycoside and peptide antibiotics

\begin{tabular}{|c|c|c|c|c|c|c|c|}
\hline \multirow[b]{2}{*}{ Treponeme strains } & \multicolumn{7}{|c|}{ Inhibitory concentrations of antimicrobial agents } \\
\hline & $\begin{array}{l}\text { Strepd } \\
(\mu g . / m l .)\end{array}$ & $\begin{array}{l}D H S M^{\mathbf{e}} \\
(\mu g . / \mathrm{ml} .)\end{array}$ & $\begin{array}{l}\operatorname{Kan}^{\mathrm{t}} \\
(\mu g . / \mathrm{ml} .)\end{array}$ & $\begin{array}{l}\text { Gents } \\
(\mu g . / m l .)\end{array}$ & $\begin{array}{l}\mathrm{Neo}^{\mathrm{h}} \\
(\mu \mathrm{g} . / \mathrm{ml} .)\end{array}$ & $\begin{array}{l}\operatorname{Viom}^{1} \\
(\mu \mathrm{g} . / \mathrm{ml} .)\end{array}$ & $\begin{array}{l}\text { Tyroj } \\
(\mu \mathrm{g} . / \mathrm{ml} .)\end{array}$ \\
\hline $\begin{array}{l}\text { Reiter } \\
\text { Kazan }^{\mathrm{a}} \\
T . \text { refringens } \\
\text { Calligyrum }^{\mathrm{b}} \\
\text { Nichols }^{\mathrm{b}} \\
\text { Microdentium FM }^{\mathrm{c}} \\
\text { Ambiguum }^{\mathrm{c}} \\
\text { T-32-A } \\
\text { T. } \text { vincentii }^{\mathrm{C}} \mathrm{N}-9\end{array}$ & $\begin{array}{r}100 \\
500 \\
10 \\
10 \\
10 \\
10 \\
100 \\
100 \\
10\end{array}$ & $\begin{array}{r}500 \\
100 \\
1 \\
1 \\
1 \\
1 \\
10 \\
100 \\
100\end{array}$ & $\begin{array}{r}1,000 \\
1,000 \\
100 \\
100 \\
1 \\
100 \\
100 \\
100 \\
100\end{array}$ & $\begin{array}{r}500 \\
\text { ND } \\
\text { ND } \\
\text { ND } \\
1 \\
10 \\
\text { ND } \\
\text { ND } \\
\text { ND }\end{array}$ & $\begin{array}{r}500 \\
500 \\
10 \\
10 \\
1 \\
10 \\
100 \\
10 \\
100\end{array}$ & $\begin{array}{r}>1,000 \\
>1,000 \\
100 \\
100 \\
10 \\
10 \\
100 \\
100 \\
1,000\end{array}$ & $\begin{array}{r}500 \\
100 \\
100 \\
10 \\
10 \\
100 \\
500 \\
100 \\
500\end{array}$ \\
\hline $\begin{array}{l}\text { HO } 5 \\
\text { HO } 18 \\
\text { HO } 27\end{array}$ & $\begin{array}{l}500 \\
500 \\
500\end{array}$ & $\begin{array}{l}100 \\
100 \\
100\end{array}$ & $\begin{array}{l}100 \\
500 \\
100\end{array}$ & $\begin{array}{l}\text { ND } \\
\text { ND } \\
\text { ND }\end{array}$ & $\begin{array}{l}500 \\
100 \\
100\end{array}$ & $\begin{array}{r}>1,000 \\
100 \\
100\end{array}$ & $\begin{array}{r}10 \\
500 \\
10\end{array}$ \\
\hline $\begin{array}{ll}\text { PF } & 23 \\
\text { PF } & 31 \\
\text { PF } & 39 \\
\text { PF } & 28 \\
\text { PF } & 44\end{array}$ & $\begin{array}{r}1,000 \\
>1,000 \\
1,000 \\
100 \\
100\end{array}$ & $\begin{array}{r}1,000 \\
>1,000 \\
1,000 \\
100 \\
100\end{array}$ & $\begin{array}{r}100 \\
500 \\
100 \\
10 \\
100\end{array}$ & $\begin{array}{l}\text { ND } \\
\text { ND } \\
\text { ND } \\
\text { ND } \\
\text { ND }\end{array}$ & $\begin{array}{l}100 \\
100 \\
500 \\
100 \\
500\end{array}$ & $\begin{array}{r}500 \\
1,000 \\
500 \\
500 \\
1,000\end{array}$ & $\begin{array}{l}100 \\
500 \\
500 \\
500 \\
500\end{array}$ \\
\hline
\end{tabular}

${ }^{\mathrm{a}} T$. phagedenis, ${ }^{\mathrm{b}} T$. refringens, ${ }^{\mathrm{c}} \boldsymbol{T}$. denticola, dstreptomycin, edihydrostreptomycin, ${ }^{\mathrm{t}}$ kanamycin, ggentamicin sulphate, ${ }^{\mathrm{h}}$ neomycin, $\mathrm{t}$ viomycin styrothricin, ND = not done.

TABLE VI Growth inhibition of treponemes by miscellaneous antibiotics and chemicals

\begin{tabular}{|c|c|c|c|c|c|c|c|}
\hline \multirow[b]{2}{*}{ Treponeme strains } & \multicolumn{7}{|c|}{ Inhibitory concentrations of antimicrobial agents } \\
\hline & $\begin{array}{l}\text { Furazd } \\
(\mu g . / m l .)\end{array}$ & $\begin{array}{l}\text { Usnic }{ }^{\mathrm{e}} \\
(\mu \mathrm{g} \cdot / \mathrm{ml} .)\end{array}$ & $\begin{array}{l}5-F U^{f} \\
(\mu g . / m l .)\end{array}$ & $\begin{array}{l}\text { Tellg } \\
(\mu \mathrm{g} \cdot / \mathrm{ml} .)\end{array}$ & $\begin{array}{l}\text { Thall } \\
(\mu g . / m l .)\end{array}$ & $\begin{array}{l}B G^{1} \\
(\mu g \cdot / \mathrm{ml} .)\end{array}$ & $\begin{array}{l}C V^{\mathrm{j}} \\
(\mu \mathrm{g} . / \mathrm{ml} .)\end{array}$ \\
\hline $\begin{array}{l}\text { Reiter }^{\mathrm{a}} \\
\text { Kazan }^{\mathrm{a}} \\
T \text {. refringens } \\
\text { Calligyrum } \\
\text { Nichols }^{\mathrm{b}} \\
\text { Microdentium FM }^{\mathrm{c}} \\
\text { Ambiguum }^{\mathrm{c}} \\
\text { T-32-A } \\
T \text {. vincentii N-9 }\end{array}$ & $\begin{array}{r}100 \\
100 \\
100 \\
100 \\
100 \\
100 \\
100 \\
100 \\
10\end{array}$ & $\begin{array}{r}1,000 \\
1,000 \\
100 \\
100 \\
100 \\
100 \\
100 \\
100 \\
500\end{array}$ & $\begin{array}{r}500 \\
1,000 \\
>1,000 \\
>1,000 \\
>1,000 \\
100 \\
>1,000 \\
100 \\
100\end{array}$ & $\begin{array}{r}100 \\
100 \\
100 \\
100 \\
100 \\
100 \\
100 \\
10 \\
10\end{array}$ & $\begin{array}{l}100 \\
500 \\
100 \\
100 \\
500 \\
500 \\
500 \\
100 \\
100\end{array}$ & $\begin{array}{l}500 \\
500 \\
100 \\
100 \\
100 \\
100 \\
100 \\
100 \\
100\end{array}$ & $\begin{array}{l}100 \\
500 \\
100 \\
500 \\
100 \\
500 \\
500 \\
500 \\
100\end{array}$ \\
\hline $\begin{array}{lr}\mathrm{HO} & 5 \\
\mathrm{HO} & 18 \\
\mathrm{HO} & 27\end{array}$ & $\begin{array}{r}10 \\
100 \\
100\end{array}$ & $\begin{array}{r}10 \\
100 \\
100\end{array}$ & $\begin{array}{r}10 \\
100 \\
100\end{array}$ & $\begin{array}{l}500 \\
100 \\
100\end{array}$ & $\begin{array}{r}10 \\
100 \\
100\end{array}$ & $\begin{array}{r}100 \\
10 \\
100\end{array}$ & $\begin{array}{l}500 \\
100 \\
100\end{array}$ \\
\hline $\begin{array}{ll}\text { PF } & 23 \\
\text { PF } & 31 \\
\text { PF } & 39 \\
\text { FP } & 28 \\
\text { PF } & 44\end{array}$ & $\begin{array}{r}500 \\
500 \\
500 \\
>1,000 \\
500\end{array}$ & $\begin{array}{r}10 \\
100 \\
10 \\
10 \\
100\end{array}$ & $\begin{array}{l}>1,000 \\
>1,000 \\
>1,000 \\
>1,000 \\
>1,000\end{array}$ & $\begin{array}{l}100 \\
500 \\
100 \\
100 \\
100\end{array}$ & $\begin{array}{l}100 \\
100 \\
100 \\
100 \\
100\end{array}$ & $\begin{array}{r}100 \\
100 \\
500 \\
10 \\
500\end{array}$ & $\begin{array}{r}100 \\
100 \\
100 \\
10 \\
100\end{array}$ \\
\hline
\end{tabular}

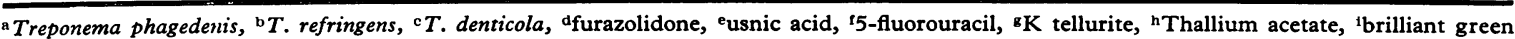
jcrystal violet.

All treponemes were resistant to cycloserine (500 to $1,000 \mu \mathrm{g} . / \mathrm{ml}$. ), polymyxin $\mathrm{B}$ sulphate (500 to $>1,000$ units $/ \mathrm{ml}$.), nitrofurazone (100 to 1,000 $\mu \mathrm{g} . / \mathrm{ml}$.), sulphathiazole $(1,000$ to $>1,000 \mu \mathrm{g} . / \mathrm{ml}$.), sulphaquinoxaline $(1,000$ to $>1,000 \mu \mathrm{g} . / \mathrm{ml}$.), sulphadiazine $(1,000$ to $>1,000 \mu \mathrm{g} . / \mathrm{ml}$.), succinyl sulphathiazole $(1,000$ to $>1,000 \mu \mathrm{g} . / \mathrm{ml}$.), nalidixic acid $(500$ tó $>1,000 \mu \mathrm{g} . / \mathrm{ml}$.), methenamine mandelate (500 to $>1,000 \mu \mathrm{g} . / \mathrm{ml}$.), 5-aminouracil $(>1,000$ $\mu \mathrm{g} . / \mathrm{ml}$.), 5-iodouracil ( $>1,000 \mu \mathrm{g} . / \mathrm{ml}$.), azocarmine ( 500 to $>1,000 \mu \mathrm{g} . / \mathrm{ml}$.), indigocarmine $(1,000$ to $>1,000 \mu \mathrm{g} . / \mathrm{ml}$.), toluidine blue $\mathrm{O}$ (500 to $>1,000$ $\mu \mathrm{g} . / \mathrm{ml}$.), lysozyme ( $>1,000 \mu \mathrm{g} . / \mathrm{ml}$.), and lysostaphin $(>1,000 \mu \mathrm{g} . / \mathrm{ml}$.).

\section{Discussion}

To interpret the data on the sensitivity of treponemes to antimicrobial agents, certain parameters have been adopted as a guide to be used in determining which antibiotic concentrations are inhibitory (Abramson, 1971). Antimicrobial agents that inhibited the growth of treponemes at $10 \mu \mathrm{g}$. or units $/ \mathrm{ml}$. of medium or below were considered to be those to which the treponemes were sensitive; however, most sensitive strains were inhibited by 1 unit or $\mu \mathrm{g} . / \mathrm{ml}$. or less. Some antibiotic concentrations obtainable in blood serum were listed by Busch and Lane (1967).

All treponemes studied were considered to be sensitive to erythromycin, bacitracin and cephalothin. 
Most strains were sensitive to the penicillins, vancomycin, tylosin, lincomycin, tetracyclines, and gentamicin. All the serum requiring isolates and the rumen fluid requiring treponemes from pig intestinal tracts were sensitive to the penicillins. Serum requiring treponemes were more sensitive to penicillin than the rumen fluid requiring intestinal strains. Strains requiring rumen fluid which were taken from the human oral cavity were generally resistant to the penicillins.

Only treponemes isolated from pig faeces were resistant to vancomycin and the tetracyclines. All other isolates were sensitive to these antibiotics. Resistance of pig faecal treponemes to tetracyclines could be due to the mixing of so-called growth stimulating levels of tetracycline antibiotics in animal feeds.

All strains studied were resistant to cycloserine, polymyxin B sulphate, nitrofurazone, sulphonamides, nalidixic acid, methenamine mandelate, 5-aminouracil, 5-iodouracil, azocarmine, indigocarmine, toluidine blue $\mathrm{O}$, lysozyme, and lysostaphin. Most strains were resistant to chloramphenicol, streptocmycin, and dihydrostreptomycin.

Growth inhibitory concentrations of antimicrobial agents for various treponemes reported in this investigation were comparable to results of previous investigators. Good agreement was generally found for penicillin (Fitzgerald and Hampp, 1952; Hampp and Fitzgerald, 1959), erythromycin (Keller and Morton, 1953; Berger, 1956; Hampp and Fitgerald, 1959) and tetracyclines (Fitzgerald and Hampp, 1952; Berger, 1956). Our results showed higher inhibitory concentrations of chloramphenicol and tyrothricin (Fitzgerald and Hampp, 1952) and polymyxin (Berger, 1956; Hampp and Fitgerald, 1959). These differences may be accounted for by our use of better anaerobic techniques than were used by previous investigators, thus allowing for rapid and heavy growth of treponemes.

\section{Summary}

The sensitivities of seventeen strains of cultivable treponemes representing most of the known species and as yet unidentified isolates from pig faeces and the human oral cavity to fifty antimicrobial agents were determined. The treponemes studied were all sensitive to cephalothin, bacitracin and erythromycin and most strains were sensitive to the penicillins, vancomycin, tylosin, lincomycin, tetracycline, and gentamicin.

All strains studied were resistant to cycloserine, polymyxin B sulphate, nitrofurazone, sulphonamides, nalidixic acid, methenamine mandelate, 5-aminouracil, 5-iodouracil, azocarmine, indigocarmine, tolui- dine blue $\mathrm{O}$, lysozyme, and lysostaphin. Most strains were resistant to chloramphenicol, streptomycin, dihydrostreptomycin, kanamycin, neomycin, viomycin, tyrothricin, furazolidone, usnic acid, 5-fluorouracil, $\mathrm{K}$ tellurite, thallium acetate, brilliant green, and crystal violet. Treponemes varied in their sensitivity to novobiocin.

This investigation was supported, in part, by the National Institutes of Health, Division of General Medical Sciences, Grant GM-14604.

\section{References}

Abramson, I. J. (1971) 'Effect of Antimicrobial Agents on Treponemes'. Thesis, Virginia Polytechnic Institute and State University, Blacksburg, Virginia 24060, U.S.A.

Anaerobe Laboratory (1970) 'Outline of Clinical Mathods in Anaerobic Bacteriology'. 2nd ed. Virginia Polytechnic Institute and State University, Blacksburg, Virginia 24060, U.S.A.

BERgER, U. (1956) Arch. Hyg. Bakt., 140, 605

- (1958a) Z. Hyg., 144, 480

(1958b) Ibid., 145, 1

(1958c) Ibid., 145, 160

(1959) Arch. Hyg. Bakt., 143, 316

(1960) Arch. klin. exp. Dermat., 210, 537

and MARggraf, H. (1960) Ibid., 210, 400

Busch, H., and LANE, M. (1967) 'Chemotherapy'. Year Book Medical Publishers, Chicago, Il'.

Collart, P., Borel, L. J., and Durel, P. (1962) Ann. inst. Pasteur., 102, 693

Dunham, W. B., Hamre, D. M., McKee, C. M., and RAKe, G. (1944) Proc. Soc. exp. Biol. (N.Y.), 55, 158

Eagle, H., and Musselman, A. (1944) f. exp. Med., 80, 493

FitzGERAld, R. J., and HAMpp, E. G. (1952) f. dent. Res., 31, 20

Hampp, E. G., and Fitzgerald, R. J. (1959) Ibid., 38, 947

Keller, R., and Morton, H. E. (1953) Amer. F. Syph., 37, 379

Mahoney, J. F., ARnold, R. C., and Harris, A. (1943) Vener. Dis. Inform., 24, 355

Nell, E. E. (1954) Amer. F. Syph., 38, 92

Nelson, R. A., JR. (1948) Amer. F. Hyg., 48, 120

Willcox, R. R. (1954) Bull. Wld Hlth Org., 10, 579

Yobs, A. R., ClaRK, J. W., JR., Mothershed, S. E., Bullard, J. C., and ARTLEY, C. W. (1968) Brit. $\mathcal{f}$. vener. Dis., 44, 116

Inhibition de la croissance des tréponèmes par les agents antimicrobiens

SOMMAIRE

On a déterminé les sensibilités, vis-à-vis de 50 agents antimicrobiens, de 17 souches de tréponèmes cultivables, représentant la plupart des espèces connues, aussi bien que de souches non identifiées isolées des fèces du porc 
et de la cavité buccale humaine. Les tréponèmes étudiés furent tous sensibles à céphalothine, bacitracine, et érythromycine, et la plupart des souches furent sensibles à pénicilline, vancomycine, tylosine, lincomycine, tétracycline et gentamicine.

Toutes les souches étudiées furent résistantes à cyclosérine, sulfate de polymyxine B, nitrofurazone, sulfamides, acide nalidixique, mandélate de méthamine, 5-amino- uracil, 5-iodo- uracil, azocarmine, indigocarmine, bleu de toluidine $\mathrm{O}$, lyzozyme et lysostaphine. La plupart des souches furent résistantes à chloramphénicol, streptomycine, dihydrostreptomycine, kanamycine, néomycine, viomycine, tyrothricine, furazolidone, acide usnique, 5fluorouracile, tellurite $\mathrm{K}$, acétate de thallium, vert brillant et cristal violet. La sensibilité à la novobiocine était variable selon la souche de tréponème considérée.

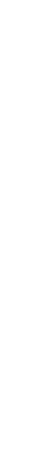

